\title{
Subtyping of Campylobacter jejuni Penner heat-stable (HS) serotype 11 isolates from human infections
}

\author{
E. SLATER and R. J. OWEN \\ Laboratory of Enteric Pathogens, Central Public Health Laboratory, 61 Colindale Avenue, London NW9 5HT
}

\begin{abstract}
High resolution molecular subtyping was applied to Campylobacter jejuni Penner heatstable (HS) 11 isolates from human infections and other sources. Strains were genotyped by restriction fragment length polymorphism analysis involving PCR-based flagellin gene (flaA) profiling with HinfI and DdeI, and pulsed-field gel electrophoretic (PFGE) profiling with SmaI and KpnI. Fla-genes of the strains were highly conserved as most $(\mathbf{9 5 \%})$ had the same fla-profile. PFGE analysis of SmaI digests was more discriminatory with 15 profile subtypes identified, although $36 \%$ of isolates had a common profile. The study showed that strains of $C$. jejuni HS11, unlike those of HS1 and the HS4 complex, were relatively homogeneous at the genomic level and that high resolution molecular techniques were essential for detailed epidemiological subtyping.
\end{abstract}

\section{Introduction}

The importance of Campylobacter jejuni as a major enteric pathogen has become established since 1977 following the development of effective selective isolation media [1]. An estimated 40000 cases of diarrhoeal disease in England and Wales are now attributed annually to infection with Campylobacter spp. [2]. The majority of speciated strains are C. jejuni and most are from sporadic cases [3].

The transmission routes of campylobacters remain unclear and are probably complex because of the involvement of various potential animal hosts and environmental reservoirs [2]. The use of phenotypic and molecular typing schemes in the laboratory provides the means for investigating the origins of such infections. For example, the Penner heat-stable (HS) serotyping scheme identifies 45 serotypes [4] within $C$. jejuni, and strains can be further subtyped by phage typing, resistotyping and various molecular techniques to provide more detailed epidemiological information $[5,6]$.

A recent 12-month study of clinical isolates of $C$. jejuni in England showed that the HS serotypes were diverse, but four (HS1, HS2, HS4 complex and HS11) currently predominated [7]. Strains of C. jejuni HS1,

Received 30 May 1997; accepted 22 August 1997. Corresponding author: Dr R. J. Owen.
HS2 and HS4 complex were genomically diverse, although some distinct clonal lines comprising isolates from mammals, chickens and cattle were identified [5, 6]. The aim of the present study was to determine diversity amongst strains of $C$. jejuni HS11, because such strains represented $c .10 \%$ of the isolates in the above study, and to assess methods for their epidemiological subtyping.

\section{Materials and methods}

\section{Bacterial strains and culture conditions}

The 78 strains of $C$. jejuni studied were from human infections (62 isolates), cattle (three isolates) and poultry (five isolates). Seven strains of unknown origin were also included. The HS11 sero-reference strain (NCTC 12510) was obtained from the National Collection of Type Cultures. All strains were cultured under micro-aerobic conditions as described previously [5]. Pure cultures were stored over liquid nitrogen $\left(-196^{\circ} \mathrm{C}\right)$ in the Cryobead system (Prolab-Diagnostics Neston, Wirral).

\section{Phenotyping}

Bacteria were identified by standard methods [8] and serotyped by the method of Penner and Hennessy [4] with a panel of 45 antisera for the Penner heat stable (HS) serotyping scheme. The antisera were provided by Dr A. Lastovica (Red Cross War Memorial Children's Hospital, Cape Town, South Africa). 


\section{Flagellin gene polymorphism}

The flaA gene was amplified by PCR with the primers and conditions described previously [9]. A single Cryobead was used as the DNA source for each sample. DNAase activity of the Lior biotype II strains was eliminated by boiling cells for $10 \mathrm{~min}$ in $30 \mathrm{ml}$ of sterile distilled water. The final PCR mix contained $5 \mathrm{ml}$ of lysate prepared from beads in a total volume of $45 \mathrm{ml}$ of PCR reagents. For DNAase negative strains, a $5-\mathrm{ml}$ cell suspension was used directly without boiling.

\section{Pulse-field gel electrophoresis (PFGE)}

Preparation of $C$. jejuni DNA for PFGE after digestion with SmaI was as described previously [10]. To digest with KpnI (Gibco BRL, Life Technologies), DNA samples in blocks were incubated in React 4 buffer for $3 \mathrm{~h}$ at $37^{\circ} \mathrm{C}$ and then re-treated with fresh enzyme and buffer for a further 3-h incubation. The PFGE conditions described previously were used for SmaI digests [10]. Modified conditions were used for Kpn I digests, in which pulsed-field times were ramped from 2 to $20 \mathrm{~s}$ with electrophoresis for $40 \mathrm{~h}$ at $200 \mathrm{~V}$. The $K p n$ I analysis was used only for strains that had identical SmaI profiles.

\section{Results}

\section{Fla-profiles}

The fla-profiling results are given in Table 1 . The majority of strains had profile H2D5 (74 of 78; 95\%), but three other profiles were observed. Profile H2D5*, which was a feature of NCTC 12510 and one field strain, differed from profile $\mathrm{H} 2 \mathrm{D} 5$ by a Dde I digest band deletion at the 350-bp position (Fig. 1). H1D4

Table 1. Summary of molecular typing results for $C$. jejuni HS11 strains

\begin{tabular}{|c|c|c|c|}
\hline Fla-profile & $\begin{array}{l}\text { PFGE (SmaI) } \\
\text { profile }\end{array}$ & $\begin{array}{l}\text { Number of } \\
\text { isolates }\end{array}$ & $\begin{array}{l}\text { PFGE (KpnI) } \\
\text { profile }\end{array}$ \\
\hline \multirow[t]{13}{*}{ H2D5 } & L1U1 & $28(36 \%)$ & $\begin{array}{l}13 \text { strains } \\
\text { (type 1: } 11 \text { strains) } \\
\text { (type 2: } 1 \text { strain) } \\
\text { (type 3: } 1 \text { strain) }\end{array}$ \\
\hline & L1U2 & $15(19 \%)$ & \\
\hline & L1U3 & $8(10 \%)$ & \\
\hline & L2U4 & 3 & \\
\hline & L1U5 & 1 & \\
\hline & L1U8 & 2 & \\
\hline & L1U9 & 4 & \\
\hline & L1U10 & 2 & \\
\hline & L1U11 & 1 & \\
\hline & L4U7 & 1 & \\
\hline & L6U2 & 2 & \\
\hline & L7U8 & 6 & \\
\hline & L8U9 & 1 & \\
\hline \multirow[t]{2}{*}{$\mathrm{H} 2 \mathrm{D} 5^{*}$} & L1U4 & $1^{\dagger}$ & \\
\hline & L1U5 & 1 & \\
\hline H1D4 & L5U3 & 1 & \\
\hline H8D6 & L3U6 & 1 & \\
\hline
\end{tabular}

${ }^{\dagger}$ NCTC 12510, sero-reference strain for HS11.

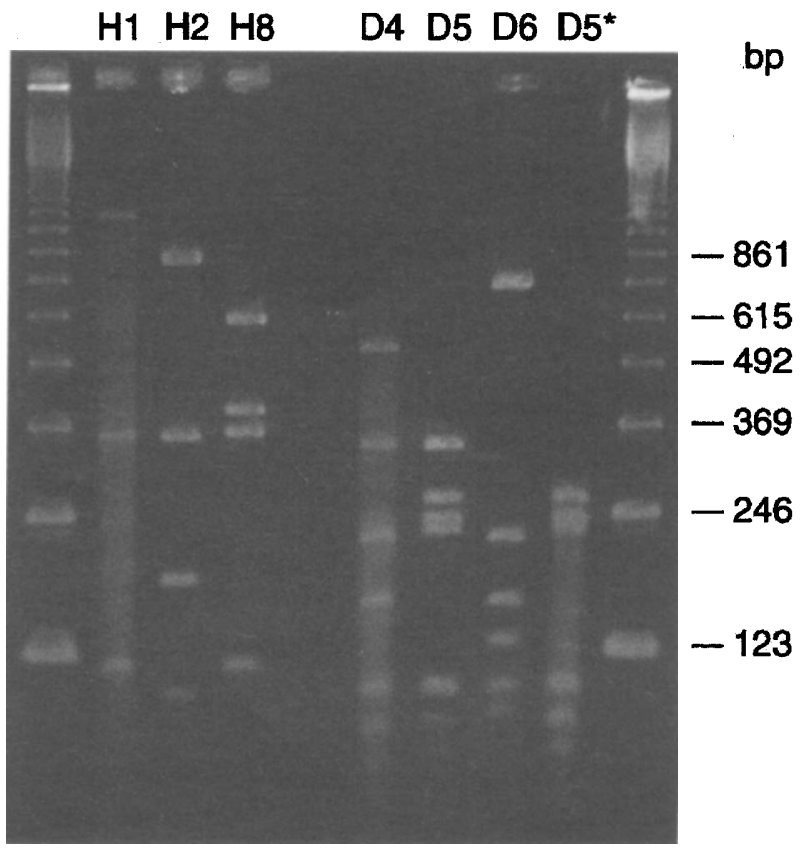

Fig. 1. Fla-type profiles digested with HinfI and DdeI for $C$. jejuni HS11 strains. Lane 1, 123-bp ladder, HinfI digests: 2, C670/93; 3, C236/93; 4, C339/93. DdeI digests: lane 5, C670/93; 6, C236/93; 7, C339/93; 8, NCTC 12510.

and H8D6 patterns were each a feature of single HS11 strains. The fla-types of isolates C670/93 (H1D4) and C627/94 (H2D5) gave total digest sizes of 200 and $650 \mathrm{bp}$ in excess of that expected for the flagellin A gene $(1.448 \mathrm{bp})$, although the PCR products were not detectably larger. Single colony picks were taken from each strain and on repeat fla-typing, both strains had the predicted total digest size.

\section{PFGE profiles}

The pulsed-field gel patterns generated with SmaI (typically six band profiles) were designated according to the upper $(\geqslant 290 \mathrm{bp}$ ) and lower $(\leqslant 235 \mathrm{bp})$ band profiles. The different types observed are shown in Fig. 2. Separation of the profiles on this basis allowed discrimination in the more variable upper band regions whilst highlighting the conserved lower band patterns. Lower band pattern 1 (L1) was seen in $67(86 \%)$ of 78 strains.

The PFGE profiles with KpnI contained more bands (typically 16) and potentially provided more discrimination than SmaI. The KpnI patterns are shown in Fig. 3. Running conditions for KpnI were optimised as several bands (five-to-nine) were present in the $\geqslant 48.5-97-b p$ region. A total run time of $40 \mathrm{~h}$ was necessary to give adequate separation. Complete digestion to avoid partials was enhanced by adding fresh $K p n \mathrm{I}$ and buffer after $3 \mathrm{~h}$ at $37^{\circ} \mathrm{C}$ followed by a further incubation for $3 \mathrm{~h}$ (J. Gibson, personal communication). 
U1 U2 U3 U4 U5 U6 U7 U8 U9 U10 U8 U11 U12 U2

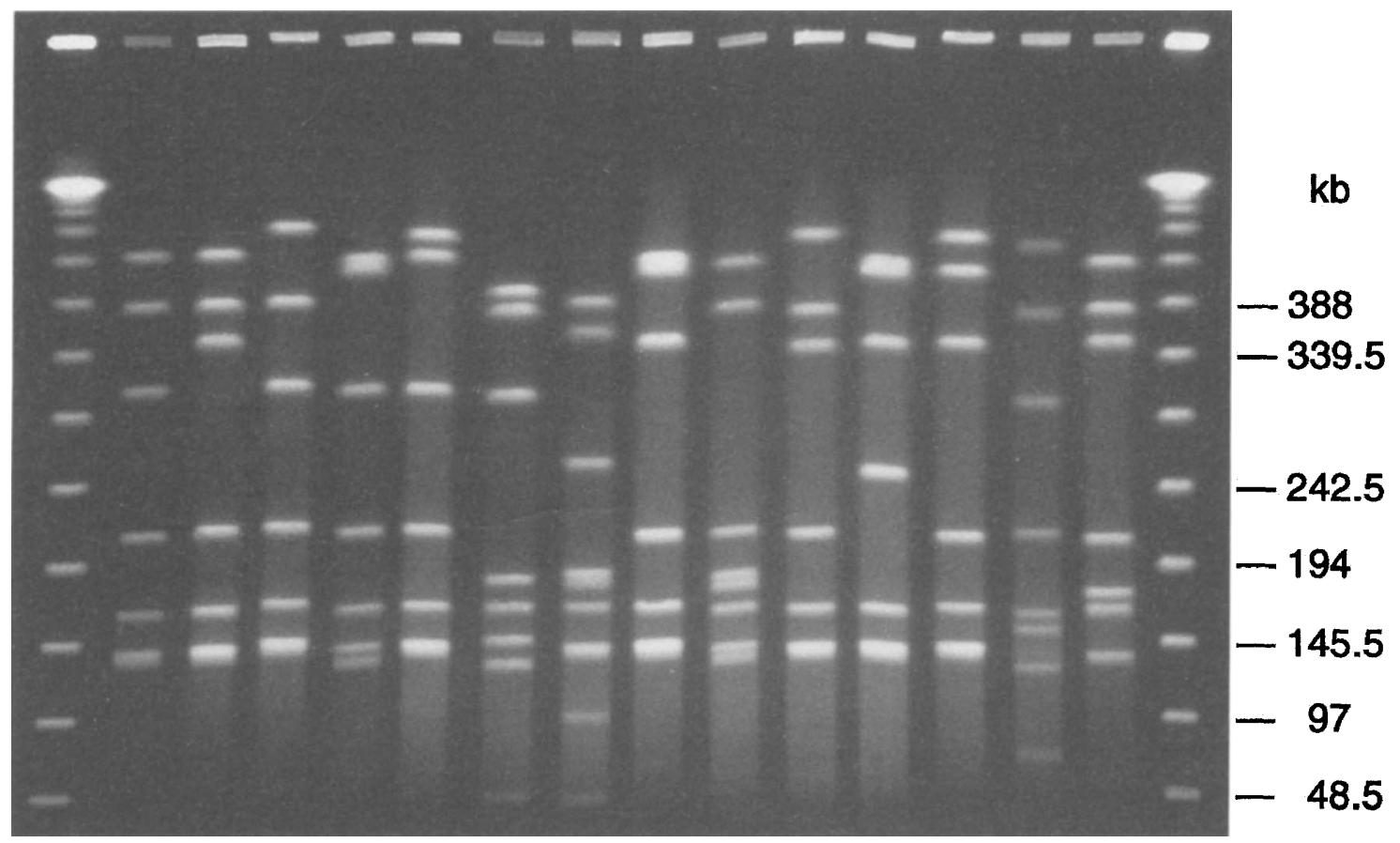

$\begin{array}{llllllllllllll}\text { L1 } & \text { L1 } & \text { L1 } & \text { L2 } & \text { L1 } & \text { L3 } & \text { L4 } & \text { L1 } & \text { L8 } & \text { L1 } & \text { L7 } & \text { L1 } & \text { L5 } & \text { L6 }\end{array}$

Fig. 2. PFGE profiles with SmaI for $C$. jejuni HS11 strains. Lane 1, lambda ladder; 2, C236/93; 3, C25/93; 4, C1160/93; 5, C1128/93; 6, C424/93; 7, C339/93; 8, C264/94; 9, C372/94; 10, C777/93; 11, C358/94; 12, C633/94; 13, C641/94; 14, C670/93; 15, C467/94; 16, lambda ladder.

Thirteen distinct PFGE profiles with SmaI were detected within the fla-type H2D5 strains (Table 1). PFGE pattern types L1U1, L1U2 and L1U3 accounted for $65 \%$ of these strains. The upper band and lower band sizes are given in Tables 2 and 3. Total genome sizes were calculated to be between 1659 and $1931 \mathrm{bp}$ with SmaI digests and between 1877 and $2096 \mathrm{bp}$ with KpnI digests.

Three different profiles were observed with KpnI among 13 strains with the same SmaI profile (Fig. 3). The seven largest bands were conserved within these strains with the differences observed in the 48.597-bp region.

Isolates from two human cases, in whom the infection had possibly been acquired abroad, had the uncommon SmaI L1U9 and L3U7 patterns. Two other human isolates from cases within the same household had a common SmaI profile (L1U1) and the same profile with Kpn I (type 1). Bovine isolates included in the study also had the common SmaI profile (L1U1). Chicken isolates had the common H2D5 fla-type but had more diverse PFGE profiles - L1U2, L1U3, L1U4, L1U10 and L5U2.

The discrimination indices for PFGE and fla-typing given in Table 4 show that the combination of flatyping with PFGE profiling did not markedly improve discrimination over PFGE profiling alone.

\section{Discussion}

Penner heat-stable serotype HS11 represents one of the commoner serotypes currently isolated from patients infected with $C$. jejuni [7]. The study of this serotype in relation to current typing methods is, therefore, important and relevant to developing an understanding of the contribution of individual serogroups to the epidemiology of $C$. jejuni infections. Fla-typing and PFGE profiling were assessed as molecular typing methods for this set of pathogenic strains.

The majority of strains $(79 \% ; 62$ of 78$)$ were isolated from sporadic cases of human illness at widely separated locations (Ashford, Belfast, Cardiff, Harrogate, Lancaster, London and Preston). It is of interest that sporadic infections with $C$. jejuni HS11 arise among a diverse human population yet strains appear to be remarkably homogeneous with respect to fla-type with $95 \%$ (74 of 78 ) strains sharing a common profile.

Strain homogeneity in serological properties and flatypes was also reflected in the similarities of PFGE profiles. Detailed analysis of the PFGE profiles showed that the largest number of band differences in SmaI digests between any two strains was five polymorphisms. Most differences $(69 \% ; 54$ of 78 ) were only one-to-two bands. Previous studies found that strains of HS1 and the HS4 complex were highly heterogeneous in their PFGE profiles, a fact consistent 


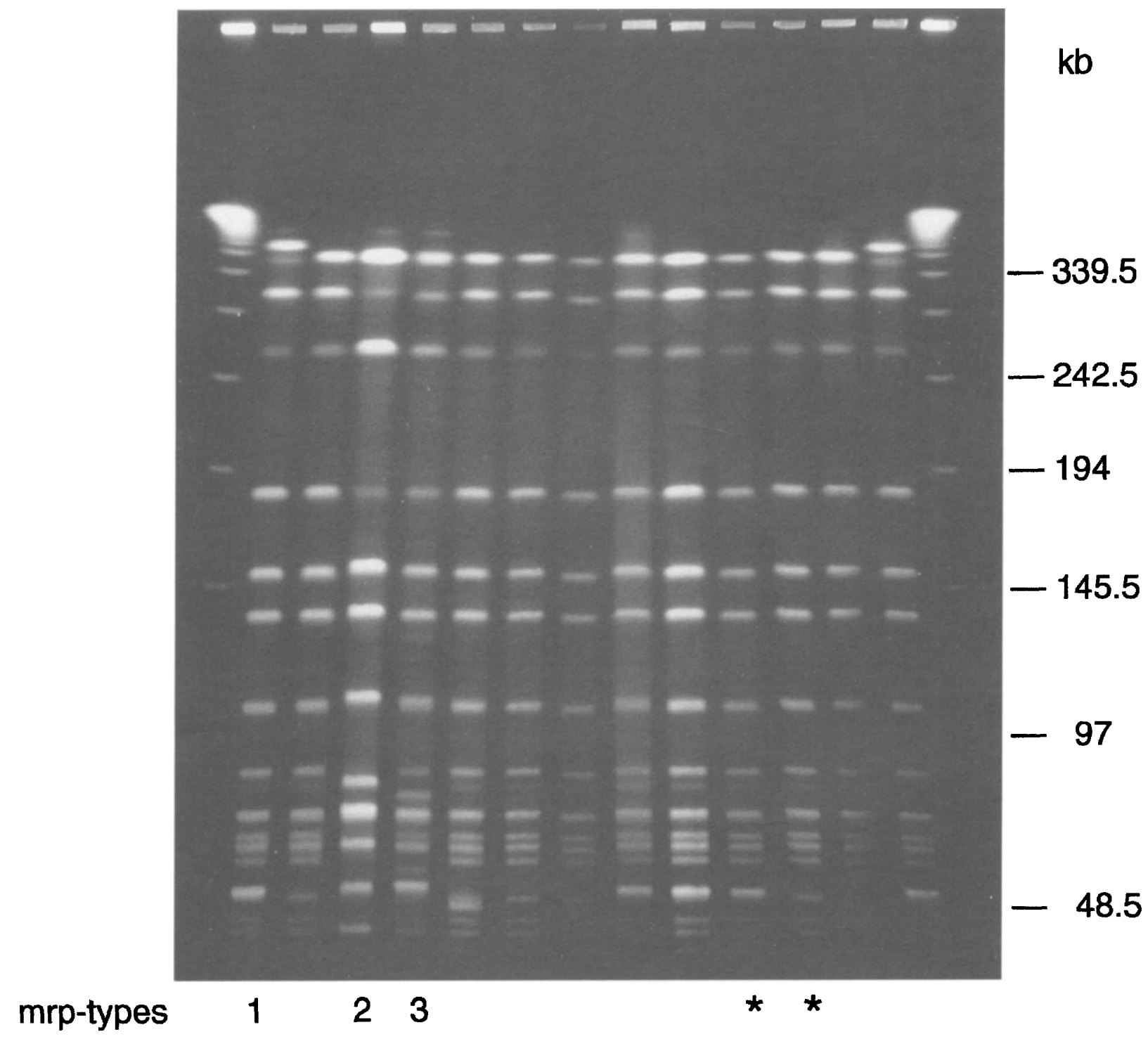

Fig. 3. PFGE profiles with KpnI for $C$. jejuni HS11 strains. Lane 1, lambda ladder; 2, C362/94; 3, C567/93; 4, C $987 / 94 ; 5, \mathrm{C} 1215 / 94 ; 6, \mathrm{C} 694 / 93 ; 7, \mathrm{C} 237 / 93 ; 8, \mathrm{C} 447 / 93 ; 9, \mathrm{C} 1122 / 93 ; 10, \mathrm{C} 1124 / 93 ; 11, \mathrm{C} 1125 / 93 ; 12$, $\mathrm{C} 752 / 93 ; 13, \mathrm{C} 28 / 94 ; 14, \mathrm{C} 363 / 94 ; 15$, lambda ladder. ${ }^{*}$, indicates from the same household.

Table 2. Sizes of lower (L) region bands in PFGE profiles (SmaI) of C. jejuni HS11 strains

\begin{tabular}{|c|c|c|c|c|c|c|c|c|c|}
\hline \multirow[b]{2}{*}{ Band no. } & \multicolumn{8}{|c|}{ Lower region bands present in profile } & \multirow[b]{2}{*}{ Size (bp) } \\
\hline & L1 & L2 & L3 & L4 & L5 & L6 & L7 & L8 & \\
\hline 1 & & & & + & & & + & & 261 \\
\hline 2 & & & + & & & & & & 220 \\
\hline 3 & + & + & & & + & + & & + & 216 \\
\hline 4 & & & & + & & & & + & 190 \\
\hline 5 & & & & + & & & & + & 181 \\
\hline 6 & & & & & & + & & & 177 \\
\hline 7 & + & + & + & + & + & + & + & + & 169 \\
\hline 8 & & & & & + & & & & 159 \\
\hline 9 & & & + & & & & & & 150 \\
\hline 10 & $t^{*}$ & + & & $t^{*}$ & & & $+^{*}$ & + & 143 \\
\hline 11 & & + & + & & & + & & + & 132 \\
\hline 12 & & & & & + & & & & 128 \\
\hline 13 & & & & + & & & & & 97 \\
\hline 14 & & & & & + & & & & 80 \\
\hline 15 & & & + & + & & & & & 52 \\
\hline
\end{tabular}


Table 3. Sizes of upper (U) region bands in PFGE profiles (SmaI) of C. jejuni HS11 strains

\begin{tabular}{|c|c|c|c|c|c|c|c|c|c|c|c|c|c|}
\hline \multirow[b]{2}{*}{ Band no. } & \multicolumn{12}{|c|}{ Upper region bands present in profile } & \multirow[b]{2}{*}{ Size $(b p)$} \\
\hline & $\mathrm{U} 1$ & $\mathrm{U} 2$ & $\mathrm{U} 3$ & $\mathrm{U} 4$ & U5 & U6 & $\mathrm{U} 7$ & U8 & U9 & U10 & U11 & $\mathrm{U} 12$ & \\
\hline 1 & & & + & & & & & & & & & & 498 \\
\hline 2 & & & & & + & & & & & + & + & + & 469 \\
\hline 3 & + & + & & + & + & & & + & + & & & & 446 \\
\hline 4 & & & & + & & & & + & & & + & & 427 \\
\hline 5 & & & & & & + & & & & & & & 404 \\
\hline 6 & + & + & + & & & + & + & & + & + & & & 385 \\
\hline 7 & & & & & & & + & & & & & & 365 \\
\hline 8 & & + & & & & & & + & & + & + & & 363 \\
\hline 9 & + & & + & + & + & & & & & & & & 317 \\
\hline 10 & & & & & & + & & & & & & & 312 \\
\hline
\end{tabular}

Table 4. Discrimination indices for $C$. jejuni typing methods applied to HS11 strains

\begin{tabular}{lccc}
\hline Method & $\begin{array}{c}\text { Number } \\
\text { of types }\end{array}$ & $\begin{array}{c}\text { Strains in } \\
\text { main type (\%) }\end{array}$ & $\begin{array}{c}\text { Discrimination } \\
\text { index [11] }\end{array}$ \\
\hline Fla-type & 4 & 95 & 0.100 \\
SmaI PFGE & 15 & 36 & 0.820 \\
Combined & 17 & 36 & 0.821 \\
\hline
\end{tabular}

with the greater number of SmaI [5] sites. The latter serotypes typically gave eight- or nine-band Sma I profiles whereas the typical HS11 SmaI profiles had only six bands. It is possible that SmaI was not the best enzyme for demonstrating diversity within the HS11 serogroup, and when KpnI was used, three different profiles were detected among 13 strains that were identical with SmaI. The running conditions were increased to $40 \mathrm{~h}$ to separate effectively the bands in the 48.5-97-bp region. Even under these conditions, 11 of the 13 strains still had the same Kpn I profile (type 1).

Tenover et al. [13] emphasised the need to include epidemiologically unrelated isolates when investigating outbreaks by PFGE to ensure that endemic strains were differentiated from outbreak strains. The results of the present study show that more precise molecular typing such as PFGE with KpnI would be needed for C. jejuni HS11 because of the inherent homogeneity of strains with that particular serotype. The extent that other HS serotypes of $C$. jejuni are conserved is not established, although data on strains representing common types in the UK, such as HS1, HS2 and HS4 complex, indicated a high level of molecular diversity $[5,6]$.

The present study found that fla-typing of $C$. jejuni HS1 1 with DdeI and HinfI provided low discrimination and even when combined with PFGE, discrimination was not significantly improved. Furthermore, the commonest fla-profile, H2D5, was diverse as it was subdivided by SmaI PFGE analysis into 13 different profile types. The results showed that the use of flatyping as a supplementary typing method to Penner serotyping was of limited use in the case of HS11 strains as it separated only $5 \%$ of isolates. SmaI and
$K p n$ I PFGE analysis would be the preferred subtyping method.

In conclusion, these results indicate that strains within serotypes of $C$. jejuni can vary significantly in genotypic homogeneity and it is necessary to optimise the choice of molecular subtyping method according to the particular serotype.

This work was funded by a grant provided by the Department of Health.

\section{References}

1. Goossens H, Wachsmuth IK. Isolation and identification of Campylobacter spp. In: Nachamkin I, Blaser MJ, Tompkins LS (eds) Campylobacter jejuni: current status and future trends. Washington, DC, ASM Press. 1992: 93-109.

2. Linton D. Old and new campylobacters. PHLS Microbiol Dig 1996; 13: 10-15.

3. Pearson AD, Healing TD. The surveillance and control of campylobacter infection. Commun Dis Rep CDR Rev 1992; 2: 133-139.

4. Penner JL, Hennessy JN. Passive hemagglutination technique for serotyping Campylobacter fetus subsp jejuni on the basis of soluble heat-stable antigens. J Clin Microbiol 1980; 12: $732-737$

5. Owen RJ, Sutherland K, Fitzgerald C, Gibson J, Borman P, Stanley J. Molecular subtyping scheme for serotypes HS1 and HS4 of Campylobacter jejuni. J Clin Microbiol 1995; 33: 872-877.

6. Gibson JR, Fitzgerald C, Owen RJ. Comparison of PFGE, ribotyping and phage-typing in the epidemiological analysis of Campylobacter jejuni serotype HS2 infections. Epidemiol Infect 1995; 115: 215-225.

7. Owen RJ, Slater E, Telford D, Donovan T, Barnham M. Subtypes of Campylobacter jejuni from sporadic cases of diarrhoeal disease at different locations of England are highly diverse. Eur J Epidemiol 1997; 13: 837-840.

8. Committee on the Microbiological Safety of Food. Interim report on Campylobacter. London, HMSO. 1993.

9. Santesteban E, Gibson J, Owen RJ. Flagellin gene profiling of Campylobacter jejuni heat stable serotype 1 and 4 complex. Res Microbiol 1996; 147: 641-649.

10. Gibson JR, Sutherland K, Owen RJ. Inhibition of DNase activity in PFGE analysis of DNA from Campylobacter jejuni. Lett Appl Microbiol 1994; 19: 357-358.

11. Hunter PR, Gaston MA. Numerical index of the discriminatory ability of typing systems: an application of Simpson's index of diversity. J Clin Microbiol 1988; 26: 2465-2466.

12. Owen RJ, Gibson JR. Update on epidemiological typing of Campylobacter. PHLS Microbiol Dig 1995; 12: 2-6.

13. Tenover FC, Arbeit RD, Goering $\mathrm{RV}$ et al. Interpreting chromosomal DNA restriction patterns produced by pulsedfield gel electrophoresis: criteria for bacterial strain typing. $J$ Clin Microbiol 1995; 33: 2233-2239. 